\title{
A Study of Software Development Team Dynamics in SPI
}

\author{
Shuib Basri ${ }^{12}$, Rory V. O’Connor ${ }^{13}$ \\ ${ }^{1}$ Lero, the Irish Software Engineering Research Centre, Ireland \\ ${ }^{2}$ Universiti Teknologi PETRONAS, Bandar Sri Iskandar, 31750 Tronoh, Perak, Malaysia \\ shuib_basri@petronas.com.my \\ ${ }^{3}$ School of Computing, Dublin City University, Ireland \\ roconnor@computing.dcu.ie
}

\begin{abstract}
The software development team is a key factor in software projects, however, achieving and maintaining positive team dynamics in software development project especially when the software companies have fewer resources in term of people, money and time is a remarkable challenge. This paper explores the dynamics of software development teams (structure, process, communication, learning and sharing) and its impact on Software Process Improvement (SPI) in very small software organization, in order to understand the impact between these two variables. We undertook a series of interviews and focus groups with very small software companies and our results show that very small companies have a high level of team dynamics although their SPI initiatives are conducted on a small scale and in an informal and indirect manner. The results also indicated that this situation occurs due to the working and social relationship, willingness to share, having a good interpersonal skill and work closely each others.
\end{abstract}

Keywords: SPI, VSEs, Team Dynamics, Grounded Theory (GT).

\section{Introduction}

Software processes are related to software development and are highly dependent on human decision making and judgement. In software development, people factors are not the only important to be consider in the process but they are also a determiner in project success [1]. People involvement in improvement activities is important because employees must adopt process innovation in their day to day activities [2]. Moreover the ways people have been treated are the important factors in organization development and change [3]. In specific, employee participation is the strongest influence on Software Process Improvement (SPI) success and, in general, peoples are the main factor in software process improvement that needs to be encouraged and support in an organization [3]. Furthermore, lack of people involvement in development activities will disturb the improvement process. Hence the aim of process improvement will be fail if people are not commit to all the propose change activities [4]. In addition the strengths and weaknesses of the current process are inside the staff hands and knowledge [5]. Hence, that even though people are the main driver for software quality but the processes has been given more attention [6]. 
Therefore in order to be success in SPI, organization must have a solid support from the software development and management team. In addition, the development and management team dynamics characteristics must exist to able to work together, share the knowledge and able to communication one another effectively. This is because the essence of software development is good relationship, effective communication and high esteem of teamwork among software development and management team in process improvement are critical in all time. This situation is become more important especially in Very Small Entities (VSEs) whose have limited resources, particularly in financial and human resources and are practicing unique processes in managing their business. Therefore, this paper aims at presenting a more comprehensive perspective of software VSEs team dynamics towards SPI initiatives. This paper is concerned with understanding VSEs issues regarding the impact of their software development team dynamics to their SPI initiatives.

\section{Background}

\subsection{Very Small Entities (VSEs)}

The definition of small and very small enterprises is challenging. To take a legalistic perspective the European Commission defines three levels of small to medium-sized enterprise (SME) as being: Small to medium - "employ fewer than 250 persons and which have an annual turnover not exceeding 50 million Euro, and/or an annual balance sheet total not exceeding 43 million Euro. The term "Very Small Entity" (VSE) had been defined by ISO/IEC 29110 as being "an entity (enterprise, organization, department or project) having up to 25 people" [8].

Micro enterprise including VSEs whose have limited resources, particularly in financial and human resources, are basically practicing unique processes in managing their business. These unique characteristics and unique situations have influenced VSEs in their business style compare to large companies [9]. In addition, these limitation and characteristics have given a big impact to companies' process infrastructures [10]. Moreover most of the management processes are performed through an informal way which most of decision-making, communication and problem solving been discussed orally and less documented. This indicates that people-oriented and communication factors are very important and significant in VSEs [11].

\subsection{Software Process Improvement (SPI)}

The primary goal of software development has changed from "conforming to plan" to "satisfy the customer - at time delivery, not a project initiation" [2]. Therefore the improvements of the development processes in order to be handle the rapidly changing environment and requirement are very significant. There are 4 
categories that could influence organizations involved in SPI namely the economic, people, organization and implementation factors [12]. Research in very small teams found that over 12 months, monthly cost and benefits have shown a positive impact of their monthly value [13]. The people factors that are related to SPI have been discussed in literature. The success of software project and process is determined by the interest of software team on the project and process itself [14]. In small software organization the influence of key individuals is a major influence [15]. However staff participation also is essential in improvement activities as they have detailed knowledge and experiences of of the current process [16].

\subsection{SPI and Teams}

In software development, human factors are not the only important to be consider in the process but they are also a determiner in project success [1]. Software development is not just creating an effective programming and tools, but also depends on people, organization and procedure. People involvement in improvement activities is important because employees must adopt process innovation in their day to day activities. The lack of involvement will disturb the improvement process because if employee did not commit themselves to all the propose change activities, the aim of process improvement will be fail [4]. Moreover, the strengths and weaknesses of the current process are inside the staff hands and knowledge [5]. Hence those people are the main factor in software process improvement that needs to be encouraged and support in an organization [2]. Moreover, [6] stated that the effect of software development team on the software product quality claimed that even though people are the main driver for software quality but the processes have been given more attention. Therefore the involvement and full commitment from teams in process improvement is critical.

The dynamic performance software project which involved many processes are always depends on the team especially in quality of communication within team. Moreover the communication may take many forms, both verbal and non-verbal [17]. Previous research shows that the level of communication in software process in depends on the size of software project [18], where they authors claim that for a small project the interaction between team members is adequate but for a larger project a mix interaction between team member and specification are required. Communication also has a related impact with the team proximity in that the increase distance from one team to another could effected the team dynamics in which it will interrupt team communication, coordination, mutual support, effort and cohesion [19]. Hence the link between team member also becoming more difficult with the increase of the team member and this will impact the team dynamics [20].

\subsection{Teams Dynamics}

Team dynamics are the hidden strengths and weakness that operate in a team between different peoples or groups. Team dynamics could effect how team reacts, behaves or performs and the effects of team dynamics are often very complex [21]. There are various forces could influence team dynamics including nature of the task, the organizational context and team composition. McCarty in her dissertation on dynamics of successful software team identified four characteristics of team 
dynamics; positive, negative, internal and external team dynamics [22]. Positive team dynamics is referred as positive forces that could lead the team to create a high performing successful team. The present of social relationship in a team could increase team productivity and enhance social and interpersonal skill [23]. Hence, the positive mode of leadership (such as well focus directive, well plan and others) in software organization could enhance the positive team dynamics [24]. Negative team dynamics is referred as negative forces that could lead the decrease of team performance and preventing people from contributes with their full potential [22]. From management point of view, in software development organization people are required three types of needs that have to be fulfilled and satisfied; social, self esteem and self-realisation needs. Internal team dynamics are referring to the forces that exist within the team itself [22]. Team member will not cooperate if they do not feel that that are a part of the team [20]. Ayman argues that within a team, roles and norms must be clear [23]. Littlepage et al. adds that cohesiveness is essential for an effective team performance and will enhance team cohesiveness [25]. A cohesive team will freely challenge each others and easily sharing new knowledge with other team members. External team dynamics are referring to the present of external forces that beyond the team control and could impact the team performance [22]. The intrinsic and extrinsic factors in projects may motivate team. Intrinsic factors are the internal factors that consist in the task and team activity itself [26]. Extrinsic factors are external factors that influence team from the outside such as reward and recognition, feedback from the organization and customer, team member pressure and the working environments.

\section{Research Study}

In order to carry out this study, a parallel approach was decided, composed of a qualitative data collection (questionnaire, interviews and focus groups) and quantitative data collection (questionnaire), with data analysis being completed separately and finally the results were merged. The overall data collection process is shown in Figure 1. We interviewed and distributed a survey questionnaire to software VSEs in Ireland. These companies were all directly involved in software product development for a variety of business domains and were determined based on researchers' personal contacts. Due to space restrictions, in this paper we only present the results from the qualitative data.

For qualitative data collection two complimentary data collection methods, (i) individual and focus group interviews, and (ii) survey questionnaire have been adopted in this study. The individual interview approach was used in this study in order to discuss the topics in depth, to get respondents' candid discussion on the topic and to be able to get the depth of information of the study situation for the research context [27]. This process followed by semi-structured interviews approach which includes the open-ended and specific questions. This approach allowed us to gather not only the information foreseen, but also unexpected type of information [28]. The respondents for the individual interview session are all software development managers / CTO / owner-directors and the focus group was with software 
development staff. The focus group interview approached was used in this study because team members develop the software and the existence team interactions helped to release inhibitions amongst the team members and are from the same company as the individual interviews participants. Focus group interviews were also chosen because it was the most appropriate method to study attitudes and experiences; to explore how opinion were constructed [29] and to understand behaviors, values and feelings, [30]. In order to gain more input and also to validate the above qualitative data for this study, we have developed and distributed a survey questionnaire to several Irish software VSEs. These companies were selected using personal contacts and were all directly involved in software product development, for a variety of business domains.

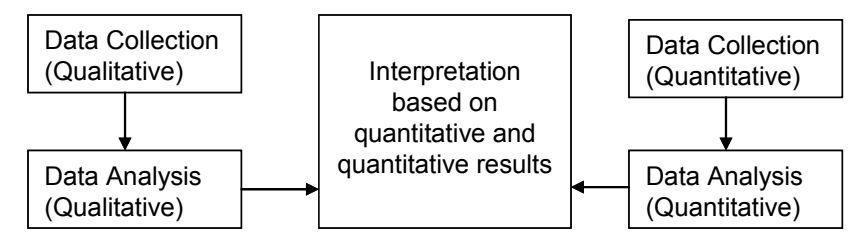

Figure 1. Research Concurrent Design- Data Collection

For the open ended data, we analyze and categories the data according to the category that this study intends to understand. The answers were group, coded and list into a table in respect to the study category issues. In overall we followed the qualitative contents analysis approach and adopted the Grounded Theory (GT) coding process in analyzing the open-ended answer [29][31]. Furthermore, in order to produce details analysis result, we have divided the survey respondents into 2 main group namely the Micro VSE (M) (1-9 employees) and Larger VSE (L) (10-25 employees).

\section{Study Findings and Discussion}

\subsection{Team Structure and Process}

From the qualitative data analysis process which adopted the GT coding approach, we consider VSEs could be divided into 2 categories; the organization and team structure category and the team process category as shown in table 1 . The organizational and team structure category indicates that due to the small number of people working in the organization, the team size is also small and this leads to a flat team and organizational structure. From the interviews analysis results indicate that all interviewees admitted that the companies have no formal team structure or a team structure only exists occasionally as maybe required for a particular project. For example, one team leader told us: 
“There are 5 developers including me and peter. No we don't have a formal team development structure at the moment, we all have the same skill and it is very flat."

Table 1. Team Structure and Process

\begin{tabular}{|c|c|c|}
\hline Sub Category & Category & Main Category \\
\hline $\begin{array}{c}\text { Organizational and Team } \\
\text { Flat Structure - }\end{array}$ & $\begin{array}{c}\text { Organizational and } \\
\text { Team Structure }\end{array}$ & $\begin{array}{l}\text { Team Structure } \\
\text { and Process }\end{array}$ \\
\cline { 1 - 1 } Team Role - informal & Team process & \\
\cline { 1 - 1 } Team Involvement - direct & & \\
\cline { 1 - 1 } Team Culture - informal & &
\end{tabular}

In additional during the analysis researchers found that due to the small number of employees, flat organization and team structure and informal environment, interviewees perceive that all people in the company are at the same level. In addition the analysis show that they have the similar level of working experience, skills and very much depends on each other in performing their task. Besides the close working space or area, high frequent and informal communication are also influences this perception. All these criteria have leaded VSEs in narrow down the gap between the management and the team development. An interview extract below best represents this situation:

"We don't have that [formal team structure] but I can see in a large company where might have that. In small company I think it is a bonus we know each other very well"

The second category is the team process category which indicates the team role, team involvement and team culture issues. The analysis shows that the staff role which includes the role in team and the task they perform in development process is very informal and very general. This could imply that the development staff could work or be assigned a different role at any time in organization development project. In addition they also can work with others or different people and different position as and when they are required. These situations have explained that team involvement process in VSEs is direct and informal in development activities. An interview extract below represents the situation describe above:

"Usually either face to face between 2 developers or over Skype with 2 developer remotely communication. In general the developers work independently and have a sole responsibility for the project. Other times they assist each other for a single project."

\subsection{Communication Process}

During the interviews sessions, the researchers have asked several questions on communication in order to understand this issue in VSEs. From the analysis, the researchers could divide the communication process in VSEs into 2 categories namely open and informal communication category and online communication category. The 
analysis also shows that the communication process in VSEs is influenced by the companies team structure and process and the working and management style as shown in table 2.

Table 2. Communication Process

\begin{tabular}{|r|r|c|}
\hline Sub Category & Category & Main Category \\
\hline \multicolumn{2}{|r|}{ Team Structure and Process } & \\
Working and Management Style & \multirow{2}{*}{ Communication Process } \\
\cline { 1 - 1 } Open Communication & Open and Informal & \\
\cline { 1 - 1 } Informal Communication & Communication & \\
\cline { 1 - 1 } Communication tools & $\begin{array}{c}\text { Online and Electronic } \\
\text { Communication }\end{array}$ & \\
\cline { 1 - 1 } Internet/ Electronically & &
\end{tabular}

In the open and informal category, the researcher has identified 24 interviews extracts that represent the category where people are more towards informal and direct/casual communication. This can be identified in the ways meeting are conducted, which are more informal, 'stand up', periodic and individual. In addition, the interviewees also agree that their day to day communication between staff is very direct and autonomous, due to the working environment in their company. This situation is confirmed by the interviewees, stating that because of the small team size that exists in the organization and the working style culture which is more toward autonomous work have create this situation. Below interview extracts below illustrate this:

"We have a formal meeting once a while but most of it is more informal. It is informal when we discuss development stuff like over the coffee. We usually share our code esp. with peter and he will look at it and share the idea. Later we will introduce to others and ask for feedback. We have informal meeting for a few minutes just to inform others regarding process before we start our tasks."

In addition the analysis also indicates the relationship between staff in the company also influences the communication process in VSEs. The analysis shows that the family and flexible environment, frequent social interaction between people and flat organization structure have given an impact on communication process in VSEs. Beside that the closeness people working space or area and high frequent of sharing activity have contribute to the communication process in VSEs. Two supporting interview extracts would be:

"I see a very open, very congenial very friendly and professional environment... I see people on the equal sourcing, openly discussing,. There no very rigid formal hierarchy. The team easily talks to management as we sit side by side.

"We work very close, meet for morning coffee. We always mix together and are very dynamic because we are small and easy to communicate each other."

The second category in this part is online and electronic communication category. From the analysis, the researchers found that the use of communication tools such as email, phone, blog, skype and internet are very active in VSEs. Such communication tools are vital to the company that has a staff member working in different locations. From the analysis researchers found that the main purpose using communication tools beside to communicate between staff members, it also the tools that could close the gap between remote and collocated staff. The analysis also indicates that the use of 
communication tools is to allow staff to share and document all work related information or knowledge in informal way. The quote explain this:

"We always skype with and other tools chat message, VPN, blog and others.

We have company internal blog to share the information among us"

\subsection{Learning and Sharing Process}

The interview data analysis elaborates how the learning and sharing process happens in VSEs. The analysis shows that the learning and sharing process main category could be detailed up into 2 important categories namely self learning category and sharing category as in table 3 .

Table 3. Learning and Sharing Process

\begin{tabular}{|c|c|c|}
\hline Sub Category & Category & Main Category \\
\hline $\begin{array}{r}\text { Com } \\
\text { Working } \\
\text { Team }\end{array}$ & $\begin{array}{l}\text { ess } \\
\text { nt Style } \\
\text { ocess }\end{array}$ & Learning and Sharing \\
\hline Training & \multirow{3}{*}{ Self Learning } & Process \\
\hline Self Learning & & \\
\hline Continuous Guidance & & \\
\hline Internal Training & \multirow{3}{*}{ Sharing } & \\
\hline Meeting & & \\
\hline Document & & \\
\hline
\end{tabular}

In the self learning category, the analysis shows in VSEs there are no formal training given to employees in enhancing their knowledge or skills. In the analysis informal training has been defined as internal training, sharing and self learning. The analysis also has explained that people in VSEs are more dependent on self learning in mastering the technology or process that is used in the organization. Besides self learning, the analysis also shows on the job training, self exploring and continues guidance from expert with in the companies are the main process that frequently been practiced in enhanced staff knowledge and skills. The following extracts are illustrative of this point.

"We haven't done any formal training but we do give our employee an opportunity to attend various courses and seminars."

"It wasn't a formal training... what I mean once you get started you could find out, who to do certain things, someone have experience can show you the way of the main resources or he can read article with your interest you want to carried out certain task. It wasn't a formal training period, I just call training because I actually learn and still learning but now is not as before"

The second category in this part is the sharing category. The analysis shows that in VSEs the knowledge sharing process happens in 3 ways: informal training, informal meetings and document sharing. Informal training happened through informal and guidance from expert, peer to peer programming process, shared books and others material, internal training, high frequent open and direct discussion with team member and online sharing with others. The informal meeting process happens through an informal stand-up meetings, direct and open discussion and online 
meetings via email and Skype. While the document sharing process have been done through note sharing and online sharing (e.g blog, email) which are informal and very personal. In relation, the analysis indicate that the learning and sharing process in VSEs is been influenced and shaped by 3 main factors which are VSEs team size and process which are small team size and flat organization structure; working and management style which are more toward autonomous work and macro management process and, communication process which are indirect and informal process. In addition from the interviews data analysis shows that in general knowledge sharing activities either via electronic or personal means are important in maintaining and evolving the current VSEs software development process. The quotes below have explained the above situations.

"However when you want to do a new things and you want to introduce a new methodology you discussed with the rest of the team, that is good and also we are supporting, if you want to do something but you not sure, you can go to any others who has more an expertise in the same area"

"We shared books and we buy books and we pass around. Generally it is informal process just asking question, grasp him and talk. Sometime we did pair programming but not always. Generally it is some kind of informal."

\subsection{SPI -Process Improvement and Assessment}

The results from the survey questionnaires have indicated that in general respondents are agreed that their software development processes rapidly change and evolve overtime. They also claimed that their development process are regularly assesses and staffs always followed or applied the latest development process method. Moreover the analysis also shown that $90 \%$ of respondents felt that their development process evolves overtime. They stated that following the best practice, client requirement, team size growth, new idea and keep up with the technology change are the reasons for the improvement and evolution of development process. The following three extracts from the open-ended questions give an indication as to how the development process have been improved and evolved with a company:

"Software process change is due to growth of the organization. We started out as 2 people 4 years ago and now have 11, so things had to change along the way"

"It will evolve as we grow in size and get more applications in production environment"

"We still do the same basic thing in software process; we change some aspects of how we work. It's a little bit ad-hoc..."

Furthermore that in question on related to the process loss issues shows that almost all or $80 \%$ of respondents' claimed that their software development processes are not affected by the process loss problem. They claimed that by using standard development tools, similar development process, having frequent guidance and mentoring activities, active in knowledge sharing and proactive coaching could avoid the process loss problems in software development process. The following extracts from the open-ended questions illustrate this situation: 
"As a manager, I don't believe in using the latest and greatest techniques for the sake of it. We'll use something that fits our team dynamics and we'll spurn something that doesn't... whether that counts."

"Our document process mostly electronically...we always sharing knowledge informally. Since this is family business, we always having informal regular meeting"

"Not really, we still do the same basic things in our software development method. We change some aspects of how we work. It's a little bit ad-hoc... Agile method... I suppose"

However the respondents also admitted that "laziness" attitudes among some staff and practicing informal and rapid changes in software development process are among the factors that could lead the process loss problem in software development process.

\section{Conclusions}

The findings indicated that respondents are agreed that their software development process frequently change and evolved over time. They also agreed that they regularly assess and update their development processes. However the finding showed that the changed and evolved processes are informal, indirect and very reactive which depends or is linked to customer requirements, developers' initiatives and technology changes. The results also indicate this situation was influence by the team structure and process is very flat and informal in VSEs. These issues have also determined the formality level of software process improvement activities in VSEs. The results also indicate that these issues also affect other critical main categories which related to VSEs software process and process. This also have create a close relationship which create a between software development team and indirectly create a high level of team dynamics and knowledge sharing activities in software development activities a shown in communication, learning and sharing category result. Beside that the external environment such as macro management style; autonomous working style active feedback from peers and management and direct involvement of management people in software activities which also have created conducive environment to the software development team in VSEs.

Furthermore the results have also shown that we found that all respondents agree that the software development team dynamics is very high. This could be identified from how the communication, relationship and learning and sharing environment status in VSEs. The results also indicated that the smaller the team in VSEs the higher level of team dynamics will be presents in the organization. In addition, the analysis also have indicate that VSEs staff have all the important criteria such as high skills, high motivated, active in sharing, direct involvement and open communication, which are important in software development process.

Acknowledgments. This work were supported, between Science Foundation Ireland grant 03/CE2/I303_1 to Lero - the Irish Software Engineering Research Centre (www.lero.ie) and Universiti Teknologi PETRONAS, Malaysia (www.utp.edu.my) 


\section{References}

1. Rosen, C.C.H., 2005 "The Influence of Intra Team relationships on the systems Development Process: A theoretical Framework of Intra-Group Dynamics.”, $17^{\text {th }}$ Workshop of the Psychology off Programming Interest Group, Sussex University,

2. Bin Basri, S and O' Connor, RV., 2010 "Organizational commitment towards software process improvement an irish software VSEs case study", Information Technology (ITSim), 2010 International Symposium, 15-17 June 2010, Kuala Lumpur,

3. Dyba, T., 2005. "An empirical investigation of the key factors for success in software process improvement". Software Engineering, IEEE Transactions, Vol.31, Issue. 5.

4. O' Connor, RV and S. Basri, 2010 "Exploring Managerial Commitment towards SPI in Small and Very Small Enterprises", In: Riel, A., et al. (eds.) EuroSPI 2010. CCIS, vol. 99, pp. 25-36. Springer, Heidelberg (2010).

5. S Basri and O' Connor, RV.,2010 "Evaluation of Knowledge Management Process in Very Small Software Companies : A Survey, Proceeding of Knowledge Management" 5th International (KMICe 2010) Conference, 25-27 May, Kuala Terengganu, Terengganu, Malaysia.

6. Beaver, J.M. and Schiavone G. A., 2006. "The effects of development team skill on software product quality". ACM SIGSOFT Software Engineering Notes, Vol. 31, No. 3.

7. European Commission, 2005, The New SME Definition: User Guide and Model Declaration available at: $\mathrm{http} /$ /europa.eu.int/comm./enterprise/enterprise policy/sme_definition/sme_user_guide.pdf. [Accessed on: 26 February 2007].

8. Laporte, C.Y.Y., Alexandre, S., and O'Connor, R. 2008. "A Software Engineering Lifecycle Standard for Very Small Enterprises", R.V.O'Connor et al (Eds) Proceedings of EuroSPI Springer-Verlag, CCIS Vol. 16, pp. 129-141.

9. Mtigwe, B., 2005. "The entrepreneurial firm internationalization process in Southern African context: A comparative approach”. International Journal of Entrepreneurial Behaviour and Research, Vol.11, Issue. 5, pp 358-377.

10. Sapovadia, V. Rajlal K., 2006 " Micro Finance: The Pillars of a Tool to Socio-Economic Development. Development Gateway", Available at SSRN: http://ssrn.com/abstract $=955062$.

11. Valtanen, A. and Sihvonen, H.M., 2008. "Employees' Motivation for SPI: Case Study in a Small Finnish Software Company". Proceeding of the $15^{\text {th }}$ European Conference, EuroSPI 2008, CCIS 16,. Springer-Verlag, pp. 152-163.

12. Hall, T., Rainer, A. and Baddoo, N. 2002, "Implementing Software Process Improvement: An empirical Study", Software Process, Improvement and Practice, Vol. 7, No 1, pp. 3-15.

13. Batisha, J. and de Figueiredo, A.D., 2000,' SPI in a Very Small Team: A Case with CMM', Software Process Improvement and Practice, Vol. 5, No. 4, pp. 243-255.

14. Komiyama T, Sunazuka T, Koyama S. 2000. "Software process assessment and improvement in NEC - current status and future direction". Software Process Improvement and Practice, Vol. 5, Issue. 1, pp. 31-43.

15. Knauber, P., Muthig, D., Schmid, K. and Widen, T., 2000. "Applying Product Line Concepts in SME”, IEEE Software, Vol. 17 No. 5, pp. 88-95.

16. Stelzer, D., Mellis, W. and Herzurm, G., 1996, "Software Process Improvement via ISO9000. Result of two surveys among the European software houses", Software Process Improvement and Practice, Vol. 2, pp 197-210 
17. Hall, T., Beecham, S., Verner, J. and Wilson, D., 2008. "The Impact of Staff turnover on Software Project: The Importance of Understanding What makes Software Practitioners Tick", Proceedings of ACM SIGMIS CPR, ACM New York, pp. 30-39.

18. Phongpaibul, M. and Boehm, B.,2005 "Improving quality through software process improvement in Thailand: initial analysis", ACM SIGSOFT Software Engineering Notes, Vol. 30, Issue 4, pp. 1-6.

19. Hoegl, M. and Proserpio, L., 2004, "Team Member Proximity and Teamwork in Innovative Projects". Research Policy, Vol. 33, No. 8, pp. 1153-1165.

20. Furumo, K. and Pearson, J.M., 2006 "An Empirical Investigation of how Trust, Cohesion and Performance Vary in Virtual and Face to Face Teams". System Sciences, Proceedings of the 39th Annual Hawaii International Conference, Vol. 1, pp. 26c- 26c .

21. Scarnati, J. T.,2001.”On becoming a team player", Team Performance Management, Vol. 7, Issue. $1 / 2$, pp. $5-10$.

22. McCarty, B., 2005. "Dynamics of a successful Team. What are the enablers and barriers to High Performing Successful Teams?" MSc Dissertation, Dublin City University.

23. Ayman, R., 2000 "Impact of team diversity on collaboration dynamics", in Collaborating across Professional Boundaries, available at http://www.stuart.iit.edu/ipro/papers/pdf/ayman.pdf. [Accessed on: 25 April 2007].

24. Singh, S. K., 2008. "Role of leadership in knowledge management: A study", Journal of Knowledge Management, Vol.12, Issue. 4, pp.3 - 15.

25. Littlepage, G.E., Cowart, L. and Kerr, B., 1989. "Relationships between Group Environment Scales and Group Performance and Cohesion", Small Group Research, Vol. 20, No. 1, pp. 50-61.

26. Kirkman, B.L., Rosen. B, Tesluk, P. E. and Gibson, C.B., 2004. "The impact of team empowerment on virtual team performance: The moderating role of face-to-face Interaction", Academy of Management Journal, Vol. 47, No 2, pp. 175-192.

27. Kvale, S., 2007. "Doing Interviews", The Sage Qualitative Research Kit, Sage

28. Li, J. Y., 2006, "Process Improvement and Risk Management in Off-the Shelf Component-Based Development", PhD Thesis, Norwegian University science and Technology.

29. Kitzinger J. 1995 "Introducing focus groups", British Medical Journal 311: 299-302.

30. Patton, M.Q, 2002 "Qualitative Evaluation and Research Methods (3nd Ed.)". Newbury Park, CA: Sage Publications, Inc.

31. Elo, S and Kyngäs, H., 2008. "The qualitative content analysis process" Journal of Advanced Nursing, Vol. 62, Issue 1, pp. $107-115$. 